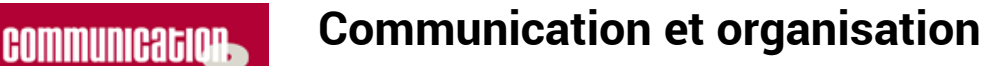

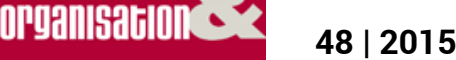

Les organisations utopiennes

Les organisations utopiennes : du rêve à la réalité...

Utopian organizations: From dream to reality...

Céline Bryon-Portet et Daniel Keller

(2) OpenEdition

Journals

Édition électronique

URL : http://journals.openedition.org/communicationorganisation/5032

DOI : 10.4000/communicationorganisation. 5032

ISSN : $1775-3546$

Éditeur

Presses universitaires de Bordeaux

Édition imprimée

Date de publication : 1 décembre 2015

Pagination : $5-10$

ISSN : 1168-5549

Référence électronique

Céline Bryon-Portet et Daniel Keller, « Les organisations utopiennes : du rêve à la réalité... »,

Communication et organisation [En ligne], 48 | 2015, mis en ligne le 01 décembre 2015, consulté le 22 septembre 2020. URL : http://journals.openedition.org/communicationorganisation/5032 ; DOI

https://doi.org/10.4000/communicationorganisation.5032

(c) Presses universitaires de Bordeaux 


\title{
Les organisations utopiennes : du rêve à la réalité...
}

\author{
Céline Brụon-Portet et Daniel Keller
}

La notion d'utopie a connu un formidable succès depuis 1516, date à laquelle Thomas More publia son célèbre ouvrage, dépeignant le gouvernement et le mode de vie des habitants d'une île imaginaire, baptisée Utopia. Nombreux furent les auteurs qui emboitèrent le pas de l'écrivain anglais et offrirent au public des récits décrivant des sociétés parfaites : Francis Bacon (La Nouvelle Atlantide), Tommaso Campanella (La Cité du Soleil), Étienne Cabet (Voyage en Icarie), et tant d'autres, se plurent ainsi à exploiter l'ambiguité du néologisme forgé par More à partir du grec latinisé « u-topia », lequel renvoie autant à un " ou topos », c'est-à-dire un « lieu de nulle part » ou un « lieu qui n'existe pas », qu'à un "eu topos », un "lieu de bonheur ». Si bien que le mot " utopie » en vint à désigner, dans le langage courant, un idéal inaccessible, et qu'au regard de cette nature chimérique, il prit une connotation parfois négative.

Pourtant, l'utopie ne demeura pas longtemps dans le giron exclusif de la littérature. Les XVIII ${ }^{\mathrm{e}}$ et $\mathrm{XIX}^{\mathrm{e}}$ siècles virent fleurir des avatars de cette notion polysémique et polymorphe, qui ne cesse de se transformer et d'offrir des visages inédits. Ainsi l'utopie se manifesta-t-elle dans l'architecture par exemple, avec la saline royale d'Arc-et-Senans, conçue par Claude Nicolas Ledoux et dont la topographie, conjuguant perfection circulaire et harmonie géométrique, devait se prolonger pour donner naissance à une cité idéale ${ }^{1}$. Elle investit également des mouvements sociopolitiques, s'incarnant dans le socialisme naissant et ses phalanstères, telle cette colonie d'inspiration fouriériste que Victor Considerant fonda au Texas en 1855, sous le nom de «La Réunion »...

La dimension utopique ne saurait donc s'épuiser dans le seul champ du rêve et de l'évasion, ce pour quoi nous préférons, pour notre part, utiliser l'adjectif «utopien » plutôt que le terme "utopique » chaque fois qu'il s'agit de qualifier un modèle de pensée et d'action alternatif, possédant une composante imaginaire mais ne revêtant pas nécessairement un caractère irréaliste. Le propre de l'utopie, en effet, n'est-il pas d'effectuer avant tout

1 Véronique Barcelo, «Comme une cité idéale... », in Éric Letonturier (dir.), Les Utopies, Paris, CNRS éditions, 2013, p.65-72. 
une contestation de l'existant, ainsi que le soulignait Karl Mannheim², et à sa suite Paul Ricœur ${ }^{3}$, lesquels lui opposaient l'idéologie, soucieuse de maintenir l'ordre établi ? Ce constat pousse d'ailleurs Raymond Ruyer à distinguer l'utopie, «description d'un monde imaginaire, en dehors de notre espace et de notre temps ", du mode utopique, envisagé comme un " exercice mental sur les possibles latéraux ", un projet normatif, « conforme non seulement à ce qui pourrait, mais ce qui devrait être $»^{4}$. Reposant sur un « principe espérance $»^{5}$, l'utopie peut pousser à l'action et contribuer à modifier le réel. Une définition relativement neutre paraît donc s'imposer :

Si l'on admet que la seule réalité concrète se confond avec ce que chaque homme vit ici et maintenant, dans le prolongement d'une histoire archivée et dans l'imminence d'un futur, à portée de volonté, l'utopie peut apparaître, dans sa plus large amplitude, comme l'ensemble des représentations et des actions, faisant référence à une autre organisation spatio-temporelle de la vie ${ }^{6}$.

Largement critiquée au siècle dernier ${ }^{7}$, notamment au regard des dérives technologiques mais surtout des totalitarismes qui furent enfantés sur le terreau d'idéaux dévoyés - comme ce fut le cas avec le communisme ${ }^{8}-$, l'utopie semble faire aujourd'hui son grand retour, dans un monde souvent qualifié de "désenchanté ", où les individus sont volontiers en quête de sens et d'espérance. De fait, on l'évoque actuellement dans des discours politiques ; des penseurs, à l'instar de Miguel Abensour', entreprennent de la réhabiliter. Quant aux éditeurs, ils multiplient à l'envi les articles et les ouvrages consacrés à cette problématique, preuve que celle-ci redevient attractive aux yeux de nombreux lecteurs ${ }^{10}$. Car d'aucuns prennent conscience

2 Karl Mannheim, Idéologie et Utopie, Paris, Marcel Rivière, 1956.

3 « Le champ des possibles s'ouvre largement au-delà de l'existant et permet d'envisager des manières de vivre radicalement autres. Ce développement de perspectives nouvelles, alternatives, définit la fonction de base de l'utopie. Ne pouvons-nous pas dire que l'imagination elle-même - à travers sa fonction utopique - a un rôle constitutif en nous aidant à repenser la nature de notre vie sociale ? N'est-ce pas par l'utopie - ce pas de côté que nous pouvons radicalement repenser ce qu'est la famille, ce qu'est la consommation, ce qu'est l'autorité, ce qu'est la religion, etc. ? ( (Paul Ricœur, L'Idéologie et l'utopie, Paris, Seuil, 1997, p. 36.)

4 Raymond Ruyer, L'Utopie et les utopies, Paris, Presses Universitaires de France, 1950, p. 9 et p. 55.

5 Ernst Bloch, Le Principe Espérance, Paris, Gallimard, 1976.

6 Jean-Jacques Wunenburger, «L'utopie, variations autour d'un mot », in Éric Letonturier (dir.), Les Utopies, Paris, CNRS éditions, « Les Essentiels d'Hermès », 2013, p. 32.

7 Voir par exemple Hans Jonas, Le Principe responsabilité, Paris, Flammarion, 2008, et Günther Anders, L'Obsolescence de l'homme, tome 2. Sur la destruction de la vie à l'époque de la troisième révolution industrielle, trad. de l'allemand par Christophe David, Paris, Fario, 2011.

8 Vladimir Boukovski, URSS : de l'utopie au désastre, Paris, Robert Laffont, 1990 ; Jacques Rossi, Quielle était belle cette utopie! Chroniques du goulag, Paris, Le Cherche-Midi, 2000.

9 Miguel Abensour, L'utopie, de Thomas More à Walter Benjamin, Paris, Sens \& Tonka, 2000 ; L'homme est un animal utopique. Utopiques II, Arles, Les Éditions de la Nuit, 2010 ; Le Procès des maîtres rêveurs. Utopiques I, Arles, Les Éditions de La Nuit, 2011.

10 Christine Bard, Georges Bertin et Lauric Guillaud (dir.), Figures de l'Utopie. Hier et aujourd'hui, Rennes, PUR, 2014 ; France Culture Papiers, n 10, été 2014, dossier spécial «Utopies. Ils rêvent d'un monde meilleur »; Norbert Elias, L'Utopie, Paris, La Découverte, 2014 ; Frédéric Rouvillois, Crime et Utopie. Une nouvelle enquête sur 
que « le renoncement au meilleur des mondes n'est nullement le renoncement à un monde meilleur " ${ }^{11}$.

Entre non-lieu et espace autre (l'hétérotopie finement étudiée par Michel Foucault), entre projet chimérique et pratique effective, récit fictif et modèle alternatif invitant à l'action, l'utopie peut être tour à tour douce mais stérile rêverie, dangereux fantasme, espérance active...

La franc-maçonnerie spéculative par exemple, à laquelle nous avons récemment consacré un ouvrage ${ }^{12}$, illustre bien cette ambivalence que porte potentiellement toute utopie. Institutionnellement née en 1717, lorsque se forma la Grande Loge de Londres et de Westminster, celle-ci remit en cause le système profondément inégalitaire de l'Ancien Régime, créant des ateliers où fraternisèrent des nobles et des roturiers, plusieurs décennies avant que la Révolution française n'abolît les privilèges. Dans l'hétérotopie de ses temples, elle ambitionnait déjà d' " améliorer à la fois l'homme et la société ", selon les mots du Rite Français dit « Groussier », largement pratiqué au Grand Orient de France. Nombreux furent d'ailleurs les initiés qui s'efforcèrent d'œuvrer « au progrès de l'humanité » au sein même de la Cité, de Victor Schœlcher à Henri Caillavet, en passant par Jules Ferry. Sous la III ${ }^{e}$ République, ils furent si actifs au plan politique, que certains gouvernements (comme celui de Léon Bourgeois) étaient composés majoritairement de francs-maçons. Cependant, la franc-maçonnerie eut aussi à lutter contre ses propres démons et connut d'indéniables dérives. Influencées notamment par les aspirations élitaires de la Stricte Observance Templière du baron Von Hund, quelques loges réintroduisirent des pratiques discriminatoires, refusant l'initiation à des juifs, des roturiers et des « sang-mêlé ». De nos jours encore, il est des obédiences qui refusent d'initier des femmes. La fraternité universelle à laquelle disent travailler les francs-maçons peut donc apparaître, dans une certaine mesure, comme un horizon dont on tente de s'approcher en vain.

Dans un domaine complètement différent, cette utopie qui se nomme «Internet ${ }^{13}$ affiche les mêmes ambiguiités : a-territorial, étant partout et nulle part à la fois, le réseau évoque d'abord une sorte de non-lieu. Mais il représente surtout un idéal de société, tant par l'originalité de sa structure réticulaire voire rhizomique, qui semble frapper d'obsolescence les organisations hiérarchiques et centralisées, que par les valeurs généreuses qu'ont insufflées ceux qui contribuèrent à le développer, sur fond de contre-culture hippie ${ }^{14}$. Espace de liberté, d'autogestion et d'échange échappant aux contrôles gouvernementaux, communauté d'égaux permettant un travail coopératif, instrument de

le nazisme, Paris, Flammarion, 2014 ; Jacques Prades, L'Utopie réaliste. Le renouveau de l'expérience coopérative, Paris, L'Harmattan, 2013 ; Éric Letonturier (dir.), Les Utopies, Paris, CNRS éditions, 2013...

11 Edgar Morin, Pour et contre Marx, Paris, Flammarion, 2012.

12 Céline Bryon-Portet et Daniel Keller, L’utopie maçonnique : améliorer l'homme et la société, Paris, Dervy, 2014.

13 Viviane Serfaty, «L'internet : fragments d'un discours utopique », Communication et langages, 1999, n 119, p.106-117.

14 Fred Turner, Aux sources de l'utopie numérique : de la contre-culture à la cyberculture, Paris, C\&F éditions, 2013. 
démocratisation ${ }^{15}$ favorisant en outre l'accès à la culture par la gratuité et le partage du savoir (logiciel libre, wikipedia...), Internet paraît réaliser le mythe du "village planétaire " de Marshall McLuhan et donner à chaque internaute le sentiment d'être ce « citoyen du monde » qu'affectionnaient les philosophes des Lumières. Pourtant, l'utopie pourrait bien tourner court et même s'inverser. Loin des desseins lumineux que forgeaient ses pionniers, la Toile se trouve progressivement investie par des individus et des groupuscules intégristes, animés par des pensées obscurantistes. Ainsi les djihadistes utilisent-ils désormais cet outil pour propager la barbarie, lançant des appels à la haine sur les sites, attirant puis endoctrinant de nouvelles recrues via les réseaux sociaux numériques, coordonnant leurs actions meurtrières grâce aux messageries, diffusant des images d'horreur, exécutions d'otages assorties de décapitations, d'immolations ou d'égorgements... Des formes violentes de communautarisme et autres repliements identitaires accompagnés de comportements fanatiques se multiplient également sur certains forums et blogs. L'activisme croissant de ceux qui utilisent paradoxalement le progrès technique pour servir des idéologies réactionnaires, prouve que le World Wide Web n'a pas réussi à relier l'humanité à elle-même et à engendrer cette « intelligence collective » espérée par Pierre Lévy, mais surtout que ténue est la frontière entre l'utopie et la contre-utopie...

Riche de sa plasticité autant que de ses contradictions internes, l'utopie ne peut manquer d'interpeller le chercheur. Les interrogations qu'elle suscite sont nombreuses et se déploient tant au plan historique (comment les formes d'expression du champ utopique évoluent-elles ?), qu'au plan sociologique (quelles structures sociales les utopies modernes et postmodernes proposentelles ? Quelles sont les motivations de ceux et de celles qui s'engagent en leur sein ?), anthropologique (quels sont les mythes, les rites et les symboles des réseaux et organisations utopiens ? Quels sont leurs significations, leurs enjeux et leurs effets ?), philosophique et politique (quelles idéologies, quels systèmes de pensée et d'organisation sous-tendent-ils ?), ou encore communicationnel (quels outils, modes et procédés d'information et de communication les entités en question mettent-elles en place ?). Raison pour laquelle ce dossier thématique a tenu à adopter une approche résolument interdisciplinaire.

Plus précisément, le présent dossier s'est donné pour objectif de réfléchir sur quelques-unes des utopies caractéristiques qui traversent la modernité et la postmodernité, à partir d'essais théoriques ou d'études de cas empiriques. Les textes rassemblés ici privilégient la présentation de ce type d'utopies "pratiquées » ou « concrètes » chers à Jean Séguy et à Ernst Bloch, et dont nous pouvons relever la présence au sein de certaines technopoles, entreprises, institutions publiques, ONG, organisations religieuses et sociétés secrètes, mais aussi à travers des mouvements sociopolitiques, des pratiques culturelles

15 Dominique Cardon, La démocratie internet : promesses et limites, Paris, Seuil, 2000. 
ou professionnelles, des réseaux numériques, des dispositifs techniques ou encore des aménagements urbains... Ils questionnent leurs enjeux et leurs finalités, leurs acteurs et leurs outils, leurs principes fondateurs, leurs discours et leur image, leurs modes d'organisation et de communication...

Florian Dauphin explore l'espace hétérotopique que constituent les catacombes de Paris et la société secrète que forment les usagers, baptisés " cataphiles». Analysant la culture propre à ces groupes marginaux, l'auteur nous entraîne ainsi dans les souterrains interdits de la Capitale. Il nous expose les valeurs, les codes sociaux et les pratiques rituelles de ceux qui sacralisent un territoire chargé d'imaginaire, considéré comme antagoniste à celui de la ville, à la surface.

Mais la ville peut, elle aussi, être le creuset dans lequel naissent des constructions utopiennes, comme le montrent Stéphane Sadoux et Julien Joanny dans leur article respectif. Le premier s'intéresse au modèle architectural et urbanistique des garden cities, conçu en Grande-Bretagne à la fin du $\mathrm{XVIII}^{\mathrm{e}}$ siècle pour échapper aux inconvénients de la ville industrielle, trop polluée, et de la campagne, trop éloignée de toutes commodités. Au-delà des aspects purement environnementaux, les projets de cités-jardins développent une dimension économique et sociale originale qui les rend attractifs, aujourd'hui encore. Le second se penche sur ces lieux culturels intermédiaires qui ouvrent des brèches au sein des villes contemporaines puis engendrent une dynamique interstitielle afin d'offrir des manières alternatives de se lier et de sorganiser : des jardins collectifs aux ZAD et aux hackerspaces, ceux-ci dépassent la simple critique de la société pour tenter d'accomplir une transformation sociale.

Mikaël Chambru, quant à lui, aborde la problématique utopienne sous un angle encore plus militant et procédural, à travers la mouvance antinucléaire. Très précisément, il s'attache à analyser les idéaux délibératifs portés et mis en place par le Réseau sortir du nucléaire, tout en pointant les paradoxes de ces pratiques démocratiques en train de se faire, qui se trouvent soumises à un cadre normatif.

Faika Ouergli, Franck Debos et Vincent Meyer, pour leur part, déplacent la question de l'utopie dans le domaine sportif. Partant du constat que les organisations sportives se réfèrent peu aux valeurs utopiennes de l'olympisme, les trois auteurs étudient la façon dont des communautés virtuelles, abritées par les réseaux sociaux numériques, réagissent, en controverse, face à ce surprenant déficit.

C'est encore dans l'espace virtuel que Christophe Duret s'immerge pour y débusquer des utopies vidéoludiques. Porteuses d'un fantasme nostalgique de démédiation, celles-ci peuvent néanmoins devenir de véritables hétérotopies lorsqu'elles sont réalisées par une communauté de joueurs. Tel est le cas des jeux de rôle goréens de Second Life, dont ce chercheur entreprend d'expliquer les différents procédés d'ouverture et de fermeture. 
Olivier Le Deuff, enfin, évoque les utopies documentaires, que le Mundaneum de Paul Otlet illustre parfaitement. Il montre notamment que les principes, les méthodes et les outils d'organisation puis de diffusion de la connaissance, reposent sur un projet politique plus vaste, qui peut devenir dystopique lorsqu'il s'engage dans le fichage des individus, par exemple.

L'ensemble de ces articles, qui montrent des facettes sensiblement différentes d'un même objet d'étude, attestent de l'incroyable diversité de l'utopie et contribueront sans doute à faire progresser l'état de la recherche autour de ce thème protéiforme. 